\title{
Evaluation d'un programme éducateur au sujet du tabagisme, de l'alcoolisme et de l'abus des drogues
}

\author{
H. Eme, P. M. Richter et G. Ritzel
}

\begin{abstract}
Résumé
Avec le programme «Consommer avec discernement» on vise à inciter les adolescents à l'abstinence ou à réduire leur consommation de tabac, l'alcool et de médicaments à l'âge, où leurs habitudes de consommation se développent. La réduction de la prédisposition à la consommation aussi bien que la réduction de la consommation elle-même sont des critères d'une intervention fructueuse. Le programme a été évalué avec une sélection représentative d'élèves de Bâle-
\end{abstract}

${ }^{1}$ Division de Médecine Sociale et Préventive de l'Université de Bâle
Ville en 8e année scolaire. L'habitude de consommation et l'attitude avant et après la présentation du programme ont été mesurées par des questions écrites standardisées. En ce qui concerne la consommation de cigarettes, un changement d'attitude et d'habitude dans la direction désirée a été relevé. La consommation d'alcool et l'attitude vis-à-vis de l'alcool n'ont pas été influencées. Si la consommation de médicaments s'est montrée stable, par contre le succès s'est manifesté par une modification de l'attitude considérable à leur égard. 